\title{
The Fine Structure of the Neck Region of Cat Spermatozoa
}

\author{
By \\ Nobuhiro SATO and Chikayoshi OURA \\ Department of Anatomy, Miyazaki Medical College, Miyazaki, 889-16, Japan \\ -Received for Publication, June 7, 1984
}

Key Words: Ultrastructure, Cat, Spermatozoa, Neck region

\begin{abstract}
Summary: The neck region of spermatozoa from the testis and the cauda epididymidis of adult cats were three dimensionally analyzed with an electron microscope. The capitulum consists of a small ventral and a large dorsal portion. The ventral portion is formed after the fusing of longitudinal striated columns 1 and 2 , while the dorsal portion, formed of $4+5+6+7$ in its uppermost end, loses 4 and 7 immediately behind the coalescent knot. The proximal centriole protrudes between 9 and $1+2$. The transverse striated columns follow along wall of the proximal centriole. The longitudinal striated columns on the ventral side tend to contact the transverse striated columns on the caudal side, while the longitudinal striated columns on the lateral sides connect with the transverse striated columns on the lateral sides. The ventral portion of the capitulum are coutinuous with the transverse striated columns on the right upper side. Triplets of the distal centriole are not seen in mature spermatozoa. The long axis of the cross section of the flattened head is not always perpendicular to the plane of the central pair of the tail axial filaments, but rotates to both sides within a range of $40^{\circ}$. Ascending toward the head, the two microtubules of the central pair lose the nexin bridge and separate, and their upper portions rotate to both sides within a range of $40^{\circ}$.
\end{abstract}

Spermatozoa have fascinated cytologists from the time they were first found by Leeuwenhoek in 1677. Subsequent to the introduction of the electron microscope and ultramicrotome, there have been many reports on the ultrastructure of mammalian spermatozoa (Blom and BirchAnderson, 1960; Burgos and Fawcett, 1955; Chakraborty, 1979; De Kretser, 1969; Fawcett, 1958, 1965, 1970, 1975; Fawcett and Ito, 1965; Fawcett and Phillips, 1969; Hancock, 1967; Iwashita and Oura, 1980; Nicander, 1962; Nicander and Bane, 1962; Olson and Linck, 1977; Pedersen, 1969, 1970, 1972; Yasuzumi et al., 1972; Zamboni and Stefanini, 1971; Zamboni et al., 1971).
All mammalian spermatozoa have much the same principal structure and a great deal has been discovered about their ultrastructure. However, the neck region of the mammalian spermatozoa needs further study because of its complexity.

The neck region has a very important function, not only as the connection between the head and the tail. but also since it is fundamentally responsible for sperm motility. However, relatively few reports using three-dimensional analysis of the ultrastructure of the neck region of mammalian spermatozoa have been published (Iwashita and Oura, 1980; Gibbons. 1963). The purpose of this study is to report an

This research was supported in part by Grants-in-Aid to the second author (C. $\bar{O})$ for Scientific Revearch from the Ministry of Education of Japan (No. 348083. No. 557009). 
analysis of the complicated structures in the neck region of mature and nearly mature spermatozoa of the cat by using serial section technique and stereography.

\section{Materials and Methods}

Adult domestic cats (Felis catus) were anesthetized; then the testis and the cauda epididymidis were excised and cut into small fragments with a razor blade in $2 \%$ glutaraldehyde solution which was adjusted to $\mathrm{pH} 7.4$ with phosphate buffer. They were fixed at $4^{\circ} \mathrm{C}$ for one hour in the same solution. After several washings in phosphate buffer, they were post-fixed in $2 \%$ osmium tetroxide for 2 hours. All fixed materials were washed in the same buffer, dehydrated in a graded series of ethanol, and embedded in Epon 812.

One micron thick sections were cut with a LKB-III type Ultrotome using glass knives, stained with $1 \%$ toluidine blue, and observed with a light microscope. Then blocks were trimmed and serial thin sections for electron microscopy were cut with glass knives and mounted on a slit of a specimen grid coated with Formvar film (Fig. 1). After being stained in LKB-2168 type Ultrostainer, they were examined with a JEM-200CX electron microscope at an acceleration voltage of $80 \mathrm{kV}$. Serial micrographs were taken of 47 spermatozoa.

In order to perform three-dimensional analysis, thicker serial sections $(0.2 \mu \mathrm{m})$ were cut with the same ultramicrotome. Each section was transferred to the center hole of a double mesh (\#100) using an eyelash probe. The sections were stained in LKB-2168 type Ultrostainer and examined with a JEM-200CX electron microscope equipped with a goniometer at an acceleration voltage of $200 \mathrm{kV}$. Seventyone spermatozoa were observed as serial stereographs.
We have defined the orientation of the neck region of the spermatozoon as shown in Figure 2. In the figure, toward the head is "cranial" and toward the tail is "caudal". Toward the peripheral doublets of No. 1 is "ventral" and toward those of No. 5 and 6 is "dorsal". The right-hand drawing is viewed from the ventral side.

The numbers of the nine peripheral doublets and nine outer dense fibers conform to the conventional system of numbering. All the pictures of the serial cross sections were adjusted so that they are exhibited from the head toward the tail.

\section{Results}

The neck region, which is short and sandwiched between the base of the head and the beginning of the middle piece, can not be delımited clearly. The cranial portion of the neck region is adjacent to the basal plate. Its caudal portion is formed by the connection of the longitudinal striated columns with the nine outer dense fibers. The neck region of the mature spermatozoa of the cat is approximately $1.0 \mu \mathrm{m}$ in length and $0.8 \mu \mathrm{m}$ in diameter.

\section{Structure of the Capitulum}

In the neck region of cat spermatozoa, immediately beneath the basal plate is the capitulum. The interspace between the basal plate and the capitulum is about $400-600 \AA$ in width.

When observing serial cross sections from the cranial portion of the neck to the basal portion of the head, the capitulum is seen as a dense mass of complicated structures (Figs. 3a-e), consisting of a small ventral and a large dorsal portion. The ventral portion, formed after the fusing of longitudinal striated columns (LSCs) 1 and 2 , is swollen and shaped like a disc. The dorsal portion, formed of $4+5+6+7$ in its uppermost end (Fig. 3b), is round on the 
dorsal articular surface but immediately behind that, 4 and 7 separate from the coalescent knot, and then the dorsal portion looks like a half moon containing two or three electron lucent spaces (Figs. $3 \mathrm{c}-\mathrm{e}$ ). In sagittal section, the dorsal portion of the capitulum is seen as funnel shaped, and its articular surface is slightly declivitous to dorso-inferior, similar to the contour of the basal plate (Fig. 4).

Structure of the Striated Columns

Among the principal structures of the connecting piece are the striated columns. In sagittal section, the longitudinal and transverse striated columns are found (Fig. 4).

Connected with the capitulum at the cranial end, the LSCs can be traced down to about $0.9-1.0 \mu \mathrm{m}$ below the basal plate, that is, to the transitional portion of the middle piece. These columns, tapering toward the caudal end, overlap the tapering cranial ends of the nine outer dense fibers of the flagellum. Stereographs of sagittal sections show clearly the overlapping connection between these two structures (Fig. 4). In cross sections of the transitional portion to the middle piece, the LSCs are found to be laterally united with each of the nine outer dense fibers (Figs. $3 g-1$ ). Further up, the LSCs unite, changing shape and reducing in number. Serial cross sections, when they are traced from the caudal portion to the cranial portion of the neck, display the manner in which the change occurs. Of the nine LSCs, 1, 2 and 9 come closer to each other, so that the spaces between 2 and 3 and between 8 and 9 are widened (Figs. 3i, j). The coalescence of 1,2 and 9 occurs on the ventral side, while 5 and 6 coalesce on the dorsal side (Figs. $3 \mathrm{~h}, \mathrm{i})$. Ascending toward the head, column 9 is seen to separate from $9+1+2$, because the proximal centriole protrudes between 9 and $1+2$ (Fig. 3e).
Transverse striated columns (TSCs) appear to run along the wall of the proximal centriole (double arrows in Figs. 3a $-f, 4$ ). In serial sections perpendicular to the proximal centriole, the TSCs are identifiable as dense matrices around the triplets (Figs. 5a-f).

Connections between the LSCs and the TSCs are found. When the nine triplets of the proximal centriole are numbered according to the methods of Pedersen (1972), the LSCs on the ventral side, such as those of 9 and 1 , contact the TSCs on the caudal side, such as those of $7,6,5,4$ and 3 , in the rather distal portion of the proximal centriole (Figs. 3e, f, 5e, f). On the other hand, the LSCs on the lateral sides, such as $3,4,7,8$ and 9 , connect with the TSCs on the lateral sides, such as $3,4,7,8$ and 9 , near the proximal boundary of the proximal centriole (Figs. 3c, d, 5a-c). The ventral portion of the capitulum connects with the TSCs 1, 2 and 3. However, the connections between the dorsal portion of the capitulum and the TSCs are not clearly seen.

Structure of the Proximal and Distal Centriole

The proximal centriole, approximately $0.5 \mu \mathrm{m} \times 0.2 \mu \mathrm{m}$. is composed of nine triplet microtubules with electron dense matrices between them. The proximal centriole declines at its base, with its long axis having an angle of $45^{\circ}$ to $60^{\circ}$ to the axis of the flagellum (Fig. 4).

A dorso-ventral view of the proximal centriole shows nine triplet microtubules scrolling centripetally clockwise (Figs. 5af). The diameter of the circle formed by the nine triplets is shorter in the distal than in the proximal portion (Figs. $5 \mathrm{a}-\mathrm{f}$ ).

The triplets of the distal centriole, which are seen in nearly mature spermatozoa from testis, are diminished and their remnants are seen in the mature spermatoxoa from 
the cauda epididymidis (Figs 3g, h). In the transitional portion between the neck and the middle piece, the doublets of the peripheral microtubules scroll centripetally clockwise just as they do in the proximal centriole. Dense matricies are found surrounding the inner subfibers (subfibers $\mathrm{A}$ ) of the doublets (Figs. 3i-l).

Cross bridges, central sheath, radial spokes and nexin bridges exist in the middle piece but they are missing in the neck region and in the transitional portion between the neck and the middle piece. The distance between the centers of the two microtubules of the central pair, being approximately $300 \AA$ in the middle piece, expands to approximately $500 \AA$ in the uppermost area (Figs. 3e, f). Two microtubules of the central pair go as far as the proximal centriole (Fig. 5b).

\section{Topographical Relationships}

When the cross sections of the head and the neck region are projected serially onto the cross section of the upper portion of the middle piece with respect to the external landmarks, the following findings are obtained:

(1) The proximal centriole protrudes between LSCs 9 and 1+2. Its long axis inclines toward doublet 9 at an angle of $10^{\circ}$ to $30^{\circ}$, when it is projected onto the uppermost cross section of the middle piece (Figs. 3e, 6).

(2) The transverse axis of the flattened head is not always parallel to a line perpendicular to the plane of the central pair but rotates to both sides within a range of $40^{\circ}$ (Fig. 6). Among 27 spermatozoa, 7 rotated clockwise, 11 rotated counterclockwise and the others were almost parallel to the line.

(3) The two microtubules of the central pair spiral at its uppermost end both clockwise and counterclockwise within a range of $40^{\circ}$, compared to the one in the transi- tional portion between the neck and the middle piece (Figs. 3e-1). Of 24 cases, 8 rotated clockwise, 6 rotated counterclockwise and 10 remained still.

\section{Discussion}

\section{Capitulum}

Fawcett and Ito (1965) observed bat spermatozoa and described that the capitulum results from the fusion of the striated columns, being triangular in longitudinal section with its base occupying about half of the implantation fossa.

On the other hand, Zamboni and Stefanini (1971), observing the mature spermatozoa of monkey, rabbit and man, described that the capitulum is transient, disappearing about the time the connecting piece attains full development. However, as shown in Figures $3 a-e$, the capitulum can be seen in mature cat spermatozoa. We also find the capitulum in mature spermatozoa of hamster and rat (Sato and Oura, unpublished). According to Iwashita and Ōura (1980), the capitulum of mature mouse spermatozoa is T-shaped, being composed of a smaller ventral portion which looks like a telephone receiver and a larger dorsal portion which is similar to a mushroom cap. In cat spermatozoa, the ventral portion of the capitulum is also smaller than the dorsal portion but the appearance is different from mouse sperm as described above. The electron lucent cavities are also found in Iwashita and Ōura's pictures of mouse spermatozoa, but the reason why the capitulum is destined to form the figure described above is still unclear.

\section{Striated Columns}

The continuities of the dense matrices around the triplets of the proximal centriole and the capitulum, and between those of the triplets and the longitudinal striated 
columns (LSCs), as reported by Fawcett and Phillips (1969), are observed in our study of cat spermatozoa. However, the manner of the continuity has not been discussed. We think these dense matrices for $m$ the transverse striated columns (TSCs) around the proximal centriole. Therefore, we understand the manner of continuity to be as follows: the LSCs on the ventral side meet the TSCs on the caudal side in the rather distal portion of the proximal centriole; the LSCs on the lateral sides have some connection with the TSCs on the lateral sides near the proximal boundary of the proximal centriole; the ventral portion of the capitulum merge with the TSCs on the right upper side; the dorsal portion of the capitulum may connect with the TSCs on the cranial side. This evidence suggests that the TSCs are joined end-toend with LSCs.

\section{Proximal and Distal Centriole}

Hancock (1967) explained that the proximal centriole was found to be a typical cylindrical structure with a dense wall and less dense center, with nine triplets set in the wall. However, we find that the diameter of the proximal centriole is shorter in the distal (ventral) than in the proximal (dorsal) boundary just as the diameter of the distal centriole is shorter in the distal (caudal) than in the proximal (cranial) boundary. We suppose that the diameter of the proximal boundary in both centrioles become wider during the spermiogenesis, because that of the proximal boundary and distal boundary are the same in early spermatid.

With their typically pinwheel appearance, the nine triplets of the proximal centriole scroll centripetally clockwise when seen from the dorsal side toward the ventral side, in other words, from the proximal to the distal end. This is the same in the distal centriole when it is observed from head to tail. From these observation we would like to emphasize that the direction of the spiral of these centrioles are the same when they are seen toward the distal end from the point where they cross each other.

There is some argument about the existence of the distal centriole in mature spermatozoa. In some reports (Fawcett and Ito, 1965; Fawcett, 1965, 1975; Fawcett and Phillips, 1969; Gordon, 1972; Woolley and Fawcett, 1973) the existence of the distal centriole in mature spermatozoa is denied, though it exists during the development of the sperm tail. Fawcett (1975) stated that only remnants of the triplet microtubules comprising the wall of the distal centriole and adhering to the inner aspect of some of the nine striated columns may occasionally be found in mature sperm. Zamboni and Stefanini (1971) assumed that the distal centriole does not disappear during sperm maturation but only undergoes profound modification.

The triplets of the distal centriole are found in nearly mature cat testicular spermatozoa in which the head chromatin has been aggregated but cytoplasm still remains around the neck and middle piece. But in mature spermatozoa from the cauda epididymidis, they are diminished and only remnants of triplets are seen.

Our observation on the mature spermatozoa of rat, monkey, mouse and hamster (not published) revealed that the remnants of the triplets and the dense matrices surround the inner subfibers of the doublets existed in rat and monkey spermatozoa just as same as in cat spermatozoa while they are diminished in mouse and hamster spermatozoa.

\section{Topographical Relationships}

Our results of observation of the site of the proximal centriole where it protrudes between the LSCs of 9 and $1+2$ are essentially identical with the serial sectional study of mouse spermatozoa by Iwashita and Oura (1980). The long axis of the proximal centriole inclines toward doublet 9 at an 
angle of $10^{\circ}$ to $30^{\circ}$ when it is projected onto the uppermost cross section of the middle piece.

In the observation of guinea pig epididymal sperm, Fawcett (1968) noticed that the transverse axis of the head is not perpendicular to the plane of the central pair, but is inclined $20-30^{\circ}$ to the plane and always in the same direction. However, in this serial section study of cat spermatozoa, a plane perpendicular to the plane of the central pair rotates not to the same direction but to both sides within a range of $40^{\circ}$. Our observation of the spermatozoa of monkey revealed much the same result (Sato and Oura, unpublished).

The two microtubules of the central pair rotates both clockwise and counterclockwise within a range of $40^{\circ}$ at the uppermost end, compared to the same structures in the transitional portion between the neck and the middle piece.

These structures are thought to have close relationships with the movement of the sperm tail. Their precise role, however, is a subject for future study.

\section{References}

1) Blom, E. and Birch-Anderson, A.: The ultrastructure of the bull sperm. I. The middle piece. Nord. Vet-Med., 12: 261-279, 1960.

2) Burgos, M. H. and Fawcett, D. W.: Studies on the fine structure of the mammalian testis. I. Differentiation of the spermatids in the cat (Felis Domestica). J. Biophysic. Biochem. Cytol., 1; 287.300, 1955.

3) Chakraborty, J.: Neck region of gerbil spermatozoa. Gamete. Res., 2: 25-34, 1979.

4) De Kretser, D. M.: Ultrastructural features of human spermiogenesis. Z. Zellforsch. mikrosk. Anat. 98: 477-505, 1969.

5) Fawcett, D. W.: The structure of the mammalian spermatozoon. Int. Rev. Cytol., 7 195-235, 1958.

6) Fawcett, D. W.: The anatomy of the mammalian spermatozoon with particular reference to the guinea pig. $Z$. Zellforsch. mikrosk.
Anat., 67 : 279-296, 1965.

7) Fawcett, D. W.: The topographical relationship between the plane of the central pair of flagellar fibrils and the transverse axis of the head in guinea-pig spermatozoa. J. Cell Sci., 3: $187-198,1968$.

8) Fawcett, D. W.: A comparative view of sperm ultrastructure. Biol. Reprod. Suppl., 2: 90$127,1970$.

9) Fawcett, D. W.: The mammalian spermatozoon. Dev. Biol., 44: 394-436, 1975.

10) Fawcett, D. W. and Ito, S.: The fine structure of bat spermatozoa. Am. J. Anat., 116: 567$610,1965$.

11) Fawcett, D. W. and Phillips, D. M.: The fine structure and development of the neck region of the mammalian spermatozoon. Anat. Rec., 165: 153-184, 1969.

12) Gibbons, I. R.: A method for obtaining serial sections of known orientation from single spermatozoa. J. Cell Biol., 16: 626-629, 1963.

13) Gordon, M.: The distal centriole in guinea pig spermiogenesis. J. Ultrastruct. Res., 39: 364-388, 1972.

14) Hancock, J. L.: The ultrastructure of mammalian spermatozoa. Advances Reprod. Physiol., 1: 125-154, 1967.

15) Iwashita, T. and Oura, C.: A three dimensional analysis of the capitellum and striated columns in the sperm neck region of the mouse. Okajimas Folia Anat. Jpn., 56: 361$382,1980$.

16) Nicander, L.: New observation on the fine structure of spermatozoa. Int. J. Fertil., 7 : 339-344, 1962.

17) Nicander, L. and Bane, A.: Fine structure of boar spermatozoa. Z. Zellforsch. mikrosk. Anat., 57: 390-405, 1962.

18) Olson, G. E. and Linck, R. W.: Observations of the structural components of flagellar axonemes and central pair microtubules from rat sperm. J. Ultrastruct. Res., 61: 21-43, 1977.

19) Pedersen, H.: Ultrastructure of the ejaculated human sperm. Z. Zellforsch. mikrosk. Anat., 94: 542-554, 1969.

20) Pedersen, H.: Observations on the axial filament complex of the human spermatozoon. J. Ultrastruct. Res., 33: 451-462, 1970.

21) Pedersen, H.: Further observations on the fine structure of the human spermatozoon. Z. Zellforsch. mikrosk. A nat., 123: 305-315, 1972.

22) Woolley, D. M. and Fawcett, D. W.: The de- 
generation and disappearance of the centrioles during the development of the rat spermatozoon. Anat. Rec., 177: 289-302, 1973.

23) Yasuzumi, G., Shiraiwa, S. and Yamamoto, $H$.: Spermatogenesis in animals as revealed by electron microscopy. XXVII. Development of the neck region and flagellum of the cat spermatozoon. Z. Zellforsch. mikrosk Anat., 125 : 497-505, 1972.

24) Zamboni, L. and Stefanini, M.: The fine structure of the neck of mammalian spermatozoa. Anat. Rec., 169: 155-172. 1971

25) Zamboni, L., Zemjanis, R. and Stefanini, M. The fine structure of monkey and human spermatozoa. Anat. Rec., 169: 129-154. 1971 . 


\section{Explanation of Figures}

\section{Plate I}

Fig. 1. Low magnification of a slit mesh on which the serial sections are mounted in order.

Fig. 2. Schematic drawing of the head and neck of the sagittal section of spermatozoa. 
Plate I
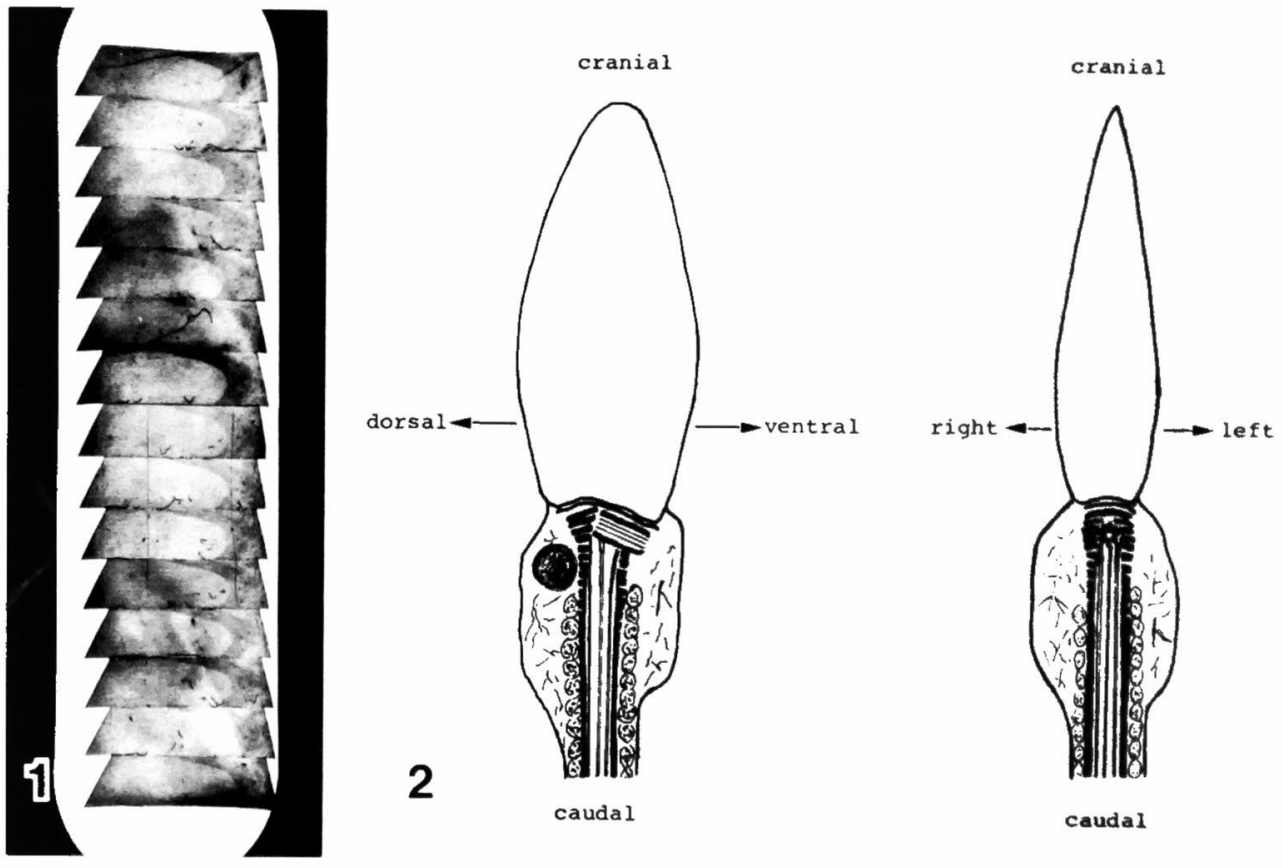


\section{Plate II and Plate III}

Fig. 3. Serial cross sections from the basal portion of the head to the transitional portion between the neck and the middle piece of a cat spermatozoon from the cauda epididymidis. Arabic numerals represent the numbers of the longitudinal striated columns by the conventional numbering system. Ascending towards the head, the longitudinal striated columns 5 and 6 coalesce to form the dorsal portion of the capitulum $(D)$, coalescing with 4 and 7 at the uppermost end on the dorsal side $(c-d)$. On the ventral side, 9,1 and 2 are coalescent, but 9 separates from $9+1+2$ at a little further up and the proximal centriole protrudes between 9 and $1+2(\mathrm{e}, \mathrm{f})$. The ventral portion of the capitulum $(V)$ is formed by $1+2$ (d). Double arrows indicate the transverse striated columns around the proximal centriole $(P)$. Doublets of the microtubules scroll centripetally clockwise (i-1), but triplets of the distal centriole are diminished and only the remnants of the triplets (single arrows) are seen. Single arrow heads indicate the connections between the capitulum and transverse striated columns while double arrow heads point out the connections between the longitudinal and transverse striated columns. $(\times 38,000)$ 

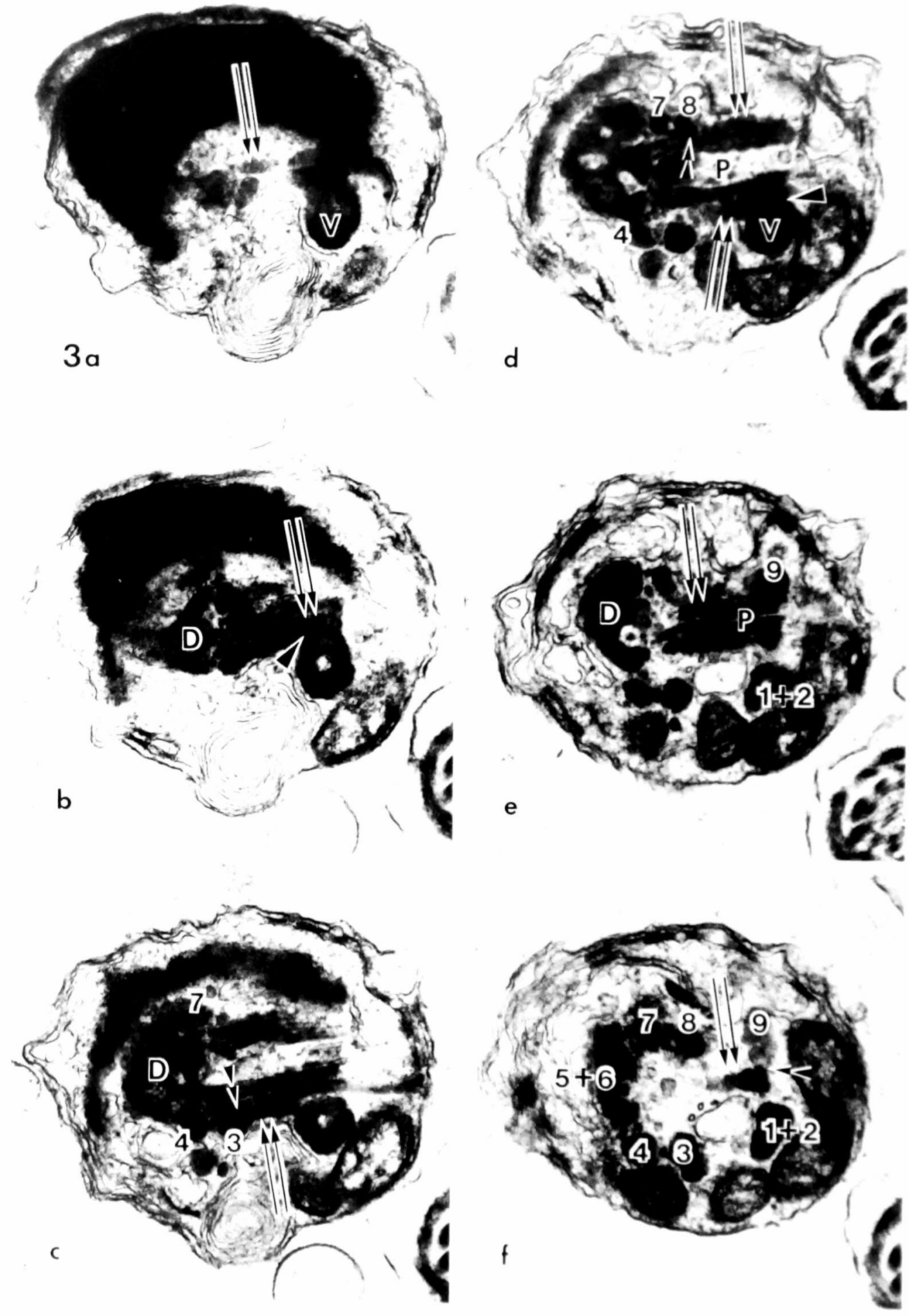
N. Sato and C. Ōura 

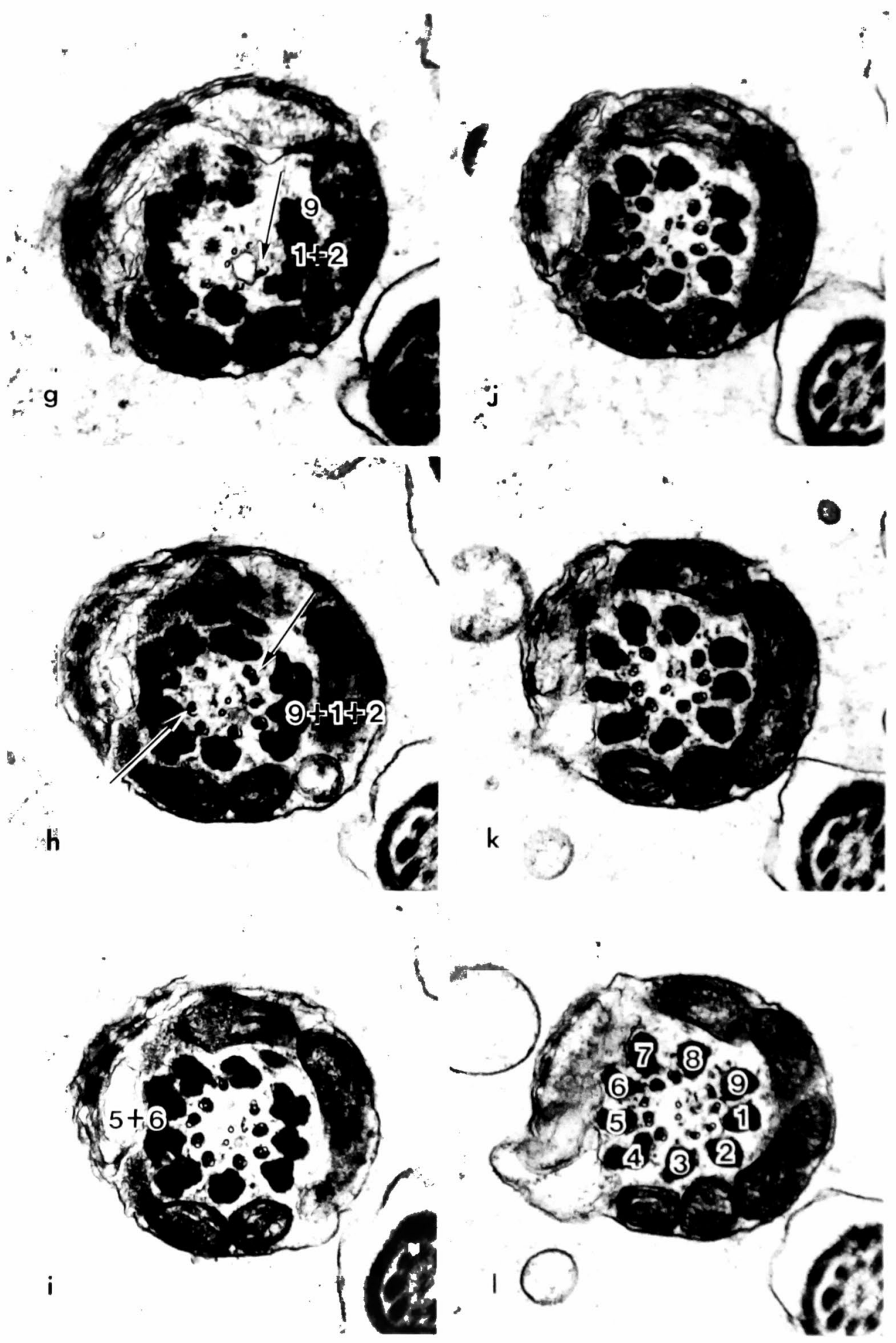


\section{Plate IV}

Fig. 4. Stereo-pair of the sagittal section of a nearly mature spermatozoon. When using a stereoscope, the section is viewed from the right side of the spermatozoon. The proximal centriole $(P)$, the dorsal portion of the capitulum $(D)$ and the transverse striated columns (double arrow) are seen. This picture shows the overlapping connection between the longitudinal striated columns (single arrow) are the nine outer dense fibers (arrow head). $(\times 34,000)$ 

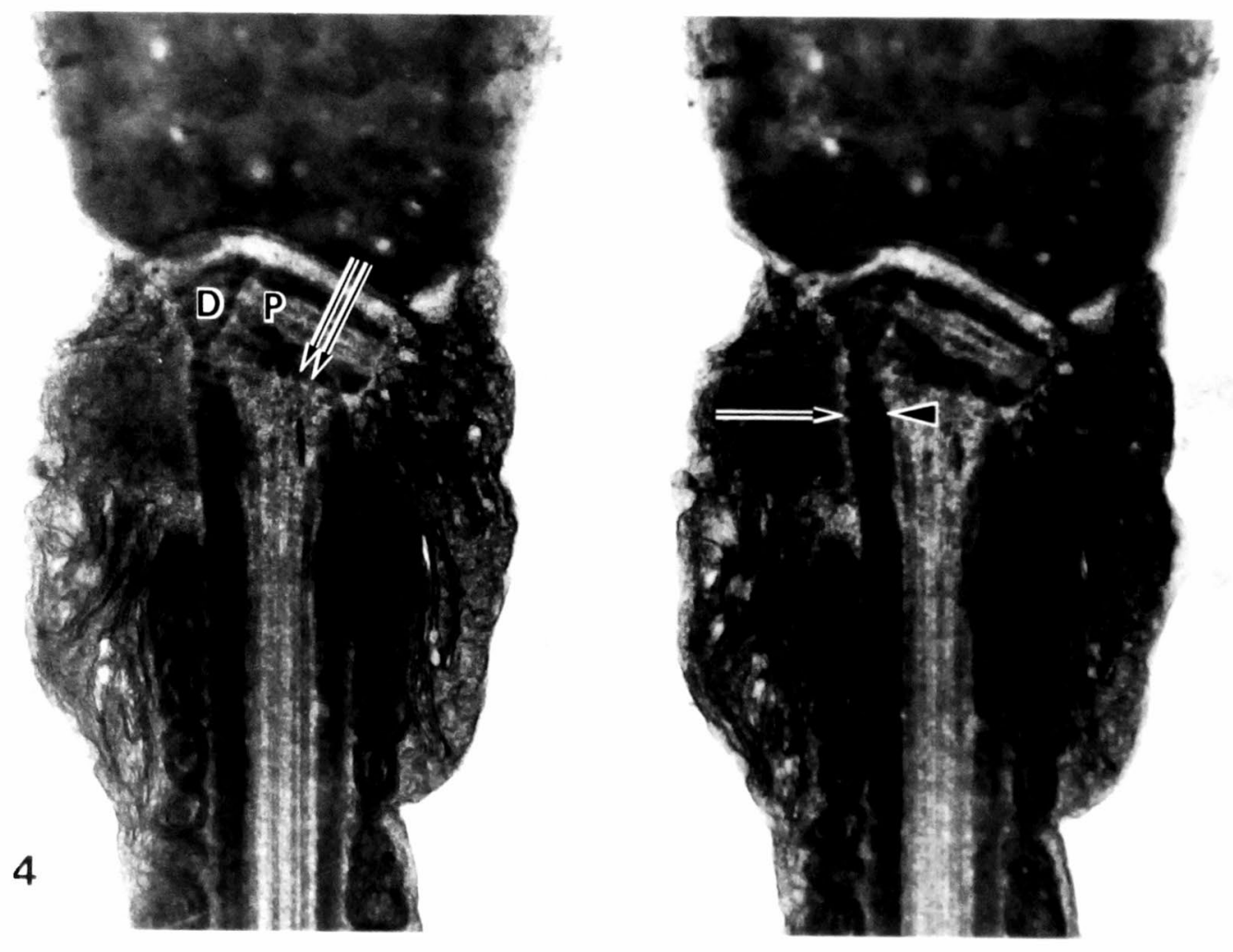


\section{Plate V}

Fig. 5. Serial sections perpendicular to the proximal centriole from the proximal portion (a) to the distal portion ( $f$ ) in a mature spermatozoon, dorso-ventral view. Black Arabic numerals represent the numbers of the triplets according to the numbering method of Pedersen (1972). White Arabic numerals represent the numbers of the longitudinal striated columns. Connections between the dense matricies around triplets and the ventral portion of the capitulum (single arrow heads) and between those of the triplets and the longitudinal striated columns (double arrow heads) are indicated. (X 52,000) 

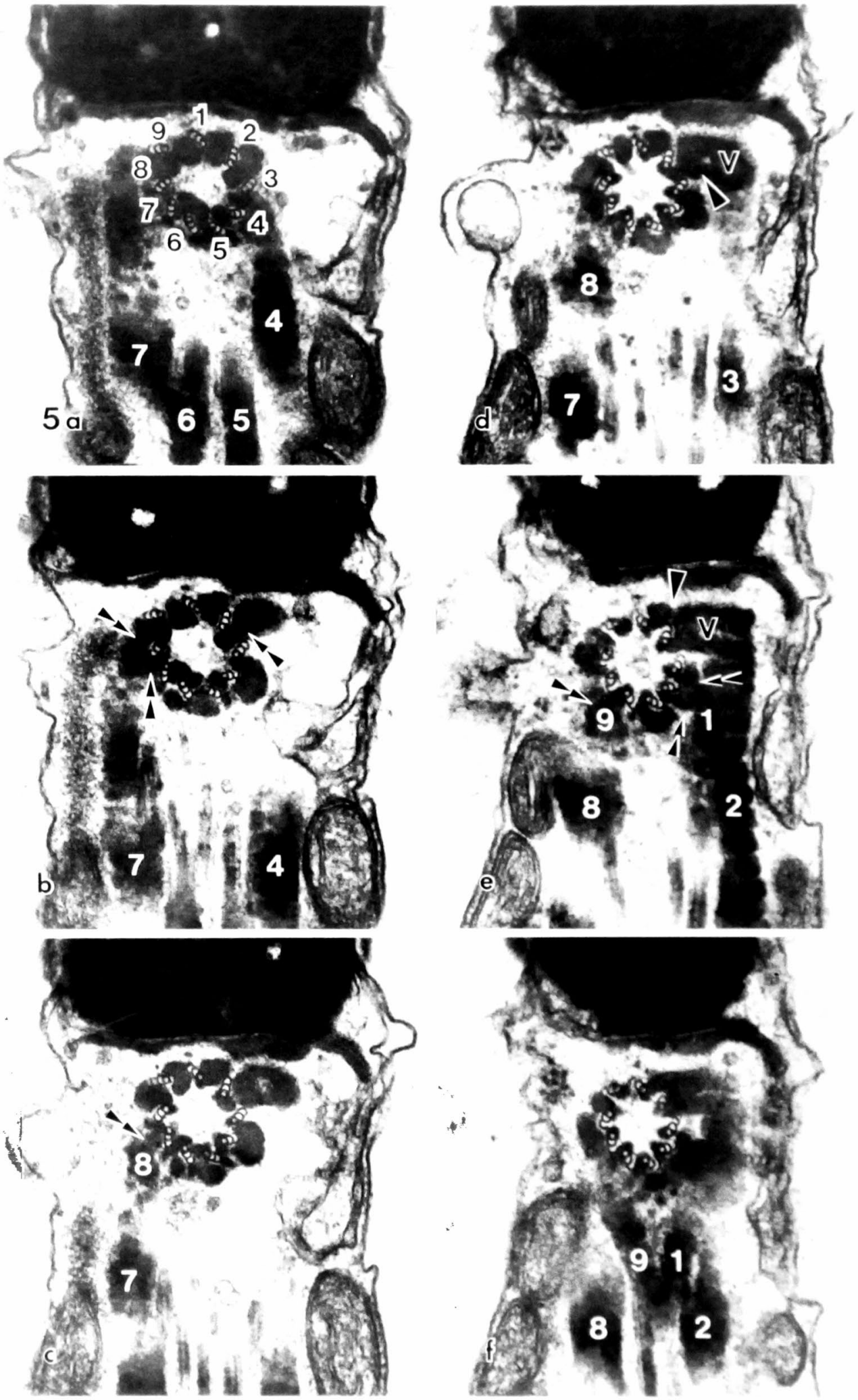


\section{Plate VI}

Fig. 6. Schematic drawing of the sagittal section of nearly mature spermatozoa. a-c: Cross sections: Their approximate level is indicated on the longitudinal section. 

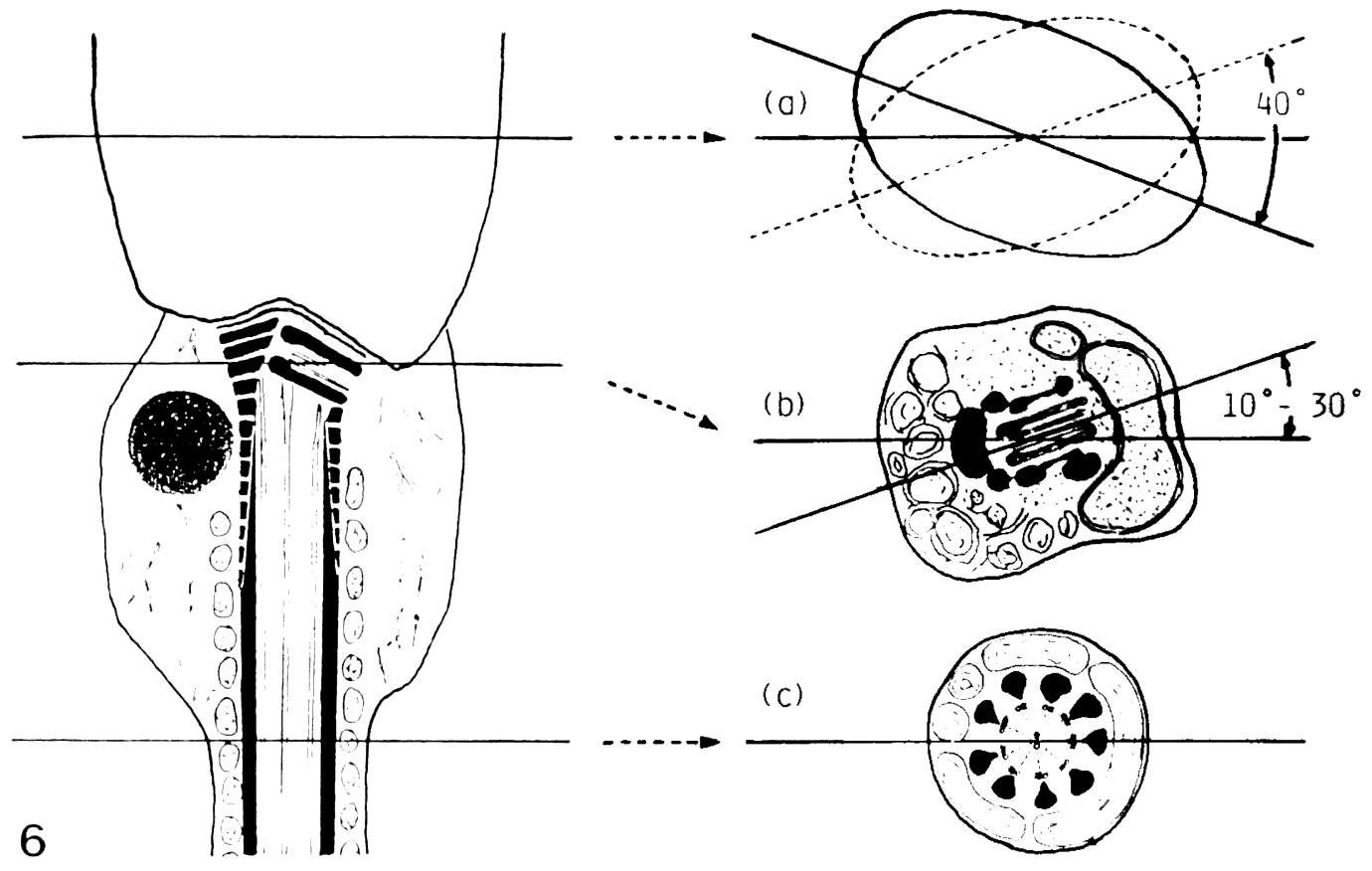